\title{
Root systems and the Erdős-Szekeres Problem
}

\author{
by
}

\author{
Roy Maltby (Burnaby, B.C.)
}

1. Introduction. An $n$-factor pure product is a polynomial which can be expressed in the form $\prod_{i=1}^{n}\left(1-x^{\alpha_{i}}\right)$ for some natural numbers $\alpha_{1}, \ldots, \alpha_{n}$. We will use two different norms, both defined in terms of the expansion of a pure product. Given a pure product $\prod_{i=1}^{n}\left(1-x^{\alpha_{i}}\right)$ whose expansion as a polynomial is $\sum_{r=0}^{\infty} a_{r} x^{r}$, the 1-norm of $\prod_{i=1}^{n}\left(1-x^{\alpha_{i}}\right)$ is denoted by $\left\|\prod_{i=1}^{n}\left(1-x^{\alpha_{i}}\right)\right\|_{1}$ and is defined to be the sum of the absolute values of the coefficients in the expansion, i.e.

$$
\left\|\sum_{r=0}^{\infty} a_{r} x^{r}\right\|_{1}:=\sum_{r=0}^{\infty}\left|a_{r}\right| .
$$

The 2-norm is defined by

$$
\left\|\sum_{r=0}^{\infty} a_{r} x^{r}\right\|_{2}:=\left(\sum_{r=0}^{\infty} a_{r}^{2}\right)^{1 / 2} .
$$

Two old problems to which norms of pure products are relevant are the Prouhet-Tarry-Escott Problem and the Erdős-Szekeres Problem. We use square brackets to delimit a list, and we call two lists (or, more precisely, $k$-lists $)\left[a_{1}, \ldots, a_{k}\right]$ and $\left[b_{1}, \ldots, b_{k}\right]$ equal if $\left(a_{1}, \ldots, a_{k}\right)$ is a permutation of $\left(b_{1}, \ldots, b_{k}\right)$. That is, a list is like a set except that repeated elements are allowed, and a list is like a tuple except that the order of entries does not matter. (Some authors call a list a multiset.) Suppose we have two unequal lists of integers $\left[a_{1}, \ldots, a_{k}\right]$ and $\left[b_{1}, \ldots, b_{k}\right]$ such that

$$
\sum_{i=1}^{k} a_{i}^{r}=\sum_{i=1}^{k} b_{i}^{r}
$$

for $r=1, \ldots, d$. Then we say that $\left[a_{1}, \ldots, a_{k}\right]$ and $\left[b_{1}, \ldots, b_{k}\right]$ form a multigrade of size $k$ and degree $d$. The Prouhet-Tarry-Escott Problem is to find

1991 Mathematics Subject Classification: 11C08, 11F22.

Key words and phrases: root system, Erdős-Szekeres Problem. 
multigrades of the smallest possible size for each degree. It is known that for any degree up to $d=9$, there is a multigrade of size $d+1$, and it is conjectured (cf. [BI94, p. 10]) that this holds for all degrees. The connection to pure products is that any $n$-factor pure product of 1 -norm $2 k$ can be used to construct a multigrade of degree $n-1$ and size $k$.

Erdős and Szekeres [ES58] asked how small the $\infty$-norm of an $n$-factor pure product can be, where the $\infty$-norm of a polynomial is defined by

$$
\left\|\sum_{i=0}^{d} a_{i} x^{i}\right\|_{\infty}:=\sup _{\{z \in \mathbb{C}:|z|=1\}}\left|\sum_{i=0}^{d} a_{i} z^{i}\right|,
$$

where $\mathbb{C}$ is the set of complex numbers and $|z|$ denotes the modulus (i.e. absolute value) of the complex number z. At the start of Section 3, we give some old inequalities relating the three different norms we have mentioned.

Throughout this paper, $\mathbb{N}$ denotes the set of all natural numbers, and $\mathbf{n}$ denotes the set $\{1, \ldots, n\}$. If $\sum_{r=0}^{\infty} a_{r} x^{r}=\prod_{i=1}^{n}\left(1-x^{\alpha_{i}}\right)$, then for each $r$,

$$
a_{r}=\mid\left\{I \subseteq \mathbf{n}: \sum_{i \in I} \alpha_{i}=r,|I| \text { even }\right\}|-|\left\{I \subseteq \mathbf{n}: \sum_{i \in I} \alpha_{i}=r,|I| \text { odd }\right\} \mid .
$$

So to make the 1 -norm of $\prod_{i=1}^{n}\left(1-x^{\alpha_{i}}\right)$ as small as possible, we need to pick the exponents $\alpha_{1}, \ldots, \alpha_{n}$ so as to maximise the number of disjoint pairs $\{I, J\}$ (that is, no two different pairs have the same $I$ or the same $J$ ) such that $\sum_{i \in I} \alpha_{i}=\sum_{j \in J} \alpha_{j}$ where $I, J \subseteq \mathbf{n},|I|$ even, and $|J|$ odd. The same criterion helps to make the 2-norm as small as possible, but one also has to consider the magnitude of individual coefficients. Among pure products having a given 1-norm, the ones with the smallest 2-norm will be those (if there are any) whose expansions have no coefficients other than 0,1 , and -1 . That is, we want to choose $\alpha_{1}, \ldots, \alpha_{n}$ so that for every $r \geq 0$, the number of ways to express $r$ as a sum of an even number of $\alpha_{1}, \ldots, \alpha_{n}$ and the number of ways to express $r$ as a sum of an odd number of $\alpha_{1}, \ldots, \alpha_{n}$ differ by at most one.

For a definition of root systems, we refer the reader to Carter [C72]. For the present, we will just describe the properties of root systems which are useful in the context of constructing pure products of small norm. Positive root systems are sets of vectors which have the property that a small number of vectors can be represented as a sum of positive roots in exactly one way, and all other vectors can be represented as a sum of an even number of positive roots in exactly as many ways as they can be represented as a sum of an odd number of positive roots. We construct pure products of small norm essentially by projecting root systems down to one dimension and using the projections of the positive roots as exponents in pure products. 
2. A construction from root systems. Let $\Pi$ be a set of fundamental roots of a root system $\Phi$. Let $\Phi^{+}$be the corresponding system of positive roots. For $r \in \Phi$, let $H_{r}$ denote the hyperplane through the origin perpendicular to $r$, and let $w_{r}$ denote the self-inverse bijection on points of the space consisting of reflection in the hyperplane $H_{r}$. The Weyl group $W$ associated with $\Phi$ is the group of bijections generated by $\left\{w_{\pi}: \pi \in \Pi\right\}$ under composition of functions. A chamber is a connected subset of $\mathcal{V} \backslash\left(\bigcup_{r \in \Phi} H_{r}\right)$ where $\mathcal{V}$ is the whole space. The intersection of the boundary of a chamber with one of the reflecting hyperplanes is called a wall of the chamber. Define

$$
S:=\frac{1}{2} \sum_{r \in \Phi^{+}} r
$$

The result that makes our construction of pure products useful is Theorem 1. Theorem 1 is essentially a well-known theorem of Weyl [C72, Theorem 10.1.8; M72, equation 0.1], which is usually expressed in the form of the equation

$$
e^{-S} \prod_{r \in \Phi^{+}}\left(1-e^{r}\right)=\sum_{w \in W}(-1)^{l(w)} e^{w(S)} .
$$

In [M96], we state and prove the interesting part of this theorem in (we believe) a clearer presentation than those in the literature. In particular, we do not mention any ring, module, Lie algebra, or [C72] "alternating element of a rational group algebra". We need one more definition before repeating our statement from [M96].

Call a chamber $C$ odd if it satisfies the following two conditions: there is exactly one $\Omega \subseteq \Phi^{+}$such that $S-\sum \Omega \in C$, and for every point $p$ in a wall of $C$, exactly half the subsets $\Omega \subseteq \Phi^{+}$such that $S-\sum \Omega=p$ have even cardinality. Theorem 1 is the interesting part of Weyl's theorem.

THEOREM 1. Every chamber is odd.

For every $v \in \mathcal{V}$, define

$$
\begin{aligned}
a(v):= & \mid\left\{\Omega \subseteq \Phi^{+}: \sum \Omega=v,|\Omega| \text { even }\right\} \mid \\
& -\mid\left\{\Omega \subseteq \Phi^{+}: \sum \Omega=v,|\Omega| \text { odd }\right\} \mid .
\end{aligned}
$$

Corollary 2. There are exactly $|W|$ points $v \in \mathcal{V}$ for which $|a(v)|=1$, and $a(v)=0$ for all other $v \in \mathcal{V}$.

Proof. This is clear from Theorem 1 since there are exactly $|W|$ chambers [C72, Corollary 2.3.3].

It is easy to see that for any natural numbers $\alpha_{1}, \ldots, \alpha_{n}$ and $a$, $\left\|\prod_{i=1}^{n}\left(1-x^{\alpha_{i}}\right)\right\|=\left\|\prod_{i=1}^{n}\left(1-x^{a \alpha_{i}}\right)\right\|$ for the 1-norm, 2-norm, and $\infty$-norm. 
Every $n$-factor pure product has 1-norm at least $2 n$. In [M94], S. Maltby answered the question: For which natural numbers $\beta_{1}, \ldots, \beta_{n}, \gamma_{1}, \ldots, \gamma_{n}$ does $\left\|\prod_{i=1}^{n}\left(1-x^{b \beta_{i}+c \gamma_{i}}\right)\right\|_{1}=2 n$ for all $b, c \in \mathbb{N}$ ? This question is equivalent to asking: In 2-dimensional space $\mathcal{V}$ with basis vectors $\pi_{1}, \pi_{2}$, for what sets of $n$ vectors $\left\{p_{1,1} \pi_{1}+p_{1,2} \pi_{2}, \ldots, p_{n, 1} \pi_{1}+p_{n, 2} \pi_{2}\right\}$ does the construction in Lemma 3 produce a pure product of 1 -norm $2 n$ for all $d_{1}, d_{2} \in \mathbb{N}$ ? S. Maltby found that, in fact, the construction produces such pure products if and only if the $p_{i, j}$ 's come from a 2 -dimensional root system.

Let $l$ be the rank of $\Phi$ and label the fundamental roots $\pi_{1}, \ldots, \pi_{l}$. Let $n=\left|\Phi^{+}\right|$and label the positive roots $r_{1}, \ldots, r_{n}$. For $i=1, \ldots, n$ and $j=$ $1, \ldots, l$, let $p_{i, j}$ be such that $\sum_{j=1}^{l} p_{i, j} \pi_{j}=r_{i}$. Since $\Pi$ is a basis of $\mathcal{V}$, the $p_{i, j}$ 's are uniquely determined. And since $\Phi^{+}$is a positive root system, the $p_{i, j}$ 's are all non-negative integers [C72, Proposition 2.1.6]. For any given $\alpha_{1}, \ldots, \alpha_{n} \in \mathbb{N}$, define for every $k \in \mathbb{N} \cup\{0\}$,

$$
b(k):=\mid\left\{I \subseteq \mathbf{n}: \sum_{i \in I} \alpha_{i}=k,|I| \text { even }\right\}|-|\left\{I \subseteq \mathbf{n}: \sum_{i \in I} \alpha_{i}=k,|I| \text { odd }\right\} \mid .
$$

It is easy to see that $\prod_{i=1}^{n}\left(1-x^{\alpha_{i}}\right)=\sum_{k=0}^{\infty} b(k) x^{k}$.

Lemma 3 is intuitively clear if one thinks of the function $p$ as a projection from the space $\mathcal{V}$ to 1 -dimensional space. Remember that $\Pi$ is a basis of $\mathcal{V}$, so $p$ in the lemma is well-defined.

Lemma 3. Let $d_{1}, \ldots, d_{l} \in \mathbb{N}$. Define $p: \mathcal{V} \rightarrow \mathbb{R}$ by

$$
p\left(\sum_{i=1}^{l} c_{i} \pi_{i}\right):=\sum_{i=1}^{l} c_{i} d_{i} .
$$

For $i=1, \ldots, n$, let $\alpha_{i}=p\left(r_{i}\right)=\sum_{j=1}^{l} p_{i, j} d_{j}$. Then for every $k \in \mathbb{N} \cup\{0\}$,

$$
b(k)=\sum_{p(v)=k} a(v) \text {. }
$$

We are now able to prove our desired result. Then

Theorem 4. Let $d_{1}, \ldots, d_{l} \in \mathbb{N}$. For $i=1, \ldots, n$, let $\alpha_{i}=\sum_{j=1}^{l} p_{i, j} d_{j}$.

$$
\left\|\prod_{i=1}^{n}\left(1-x^{\alpha_{i}}\right)\right\|_{1} \leq|W|
$$

where $W$ is the Weyl group of $\Phi$.

Proof. We have

$$
\left\|\prod_{i=1}^{n}\left(1-x^{\alpha_{i}}\right)\right\|_{1}=\left\|\sum_{k=0}^{\infty} b(k) x^{k}\right\|_{1}=\sum_{k=0}^{\infty}|b(k)| .
$$

Since $a(v)=0$ whenever $p(v) \notin \mathbb{N} \cup\{0\}$, Lemma 3 tells us that 


$$
\sum_{k=0}^{\infty}|b(k)|=\sum_{k=0}^{\infty}\left|\sum_{p(v)=k} a(v)\right| \leq \sum_{k=0}^{\infty} \sum_{p(v)=k}|a(v)|=\sum_{v \in \mathcal{V}}|a(v)| .
$$

And Corollary 2 tells us that

$$
\sum_{v \in \mathcal{V}}|a(v)|=|W|
$$

There is a reason why one could allow the $d_{j}$ 's in Theorem 4 to be any integers (not necessarily positive) which do not yield any $\alpha_{i}=0$, but there is another reason why the resulting new permitted values of $d_{j}$ 's would be redundant. Suppose one allows polynomials to include terms with negative exponents, and suppose also that one adjusts the definition of the 1-norm and 2-norm to include coefficients of negative exponents the same as coefficients of positive ones. Then, for any natural numbers $\alpha_{1}, \ldots, \alpha_{n}$, it is easy to see that the following equalities hold for the 1-norm, the 2-norm, and the $\infty$-norm:

$$
\begin{aligned}
\left\|\left(1-x^{-\alpha_{1}}\right) \prod_{i=2}^{n}\left(1-x^{\alpha_{i}}\right)\right\| & =\left\|x^{-\alpha_{1}}\left(x^{\alpha_{1}}-1\right) \prod_{i=2}^{n}\left(1-x^{\alpha_{i}}\right)\right\| \\
& =\left\|-x^{-\alpha_{1}} \prod_{i=1}^{n}\left(1-x^{\alpha_{i}}\right)\right\|=\left\|\prod_{i=1}^{n}\left(1-x^{\alpha_{i}}\right)\right\| .
\end{aligned}
$$

From this we see that if we allow negative exponents in the factors of a pure product, we can replace them with their absolute values and the expansion will have the same coefficients, but associated with different exponents, and perhaps with reversed signs, so that the norms are not affected. Because of this, it would make sense to allow the $d_{j}$ 's to be any integers not yielding any $\alpha_{i}=0$. The resulting pure product $\prod_{i=1}^{n}\left(1-x^{\alpha_{i}}\right)$ would be equivalent, for our purposes, to the pure product $\prod_{i=1}^{n}\left(1-x^{\left|\alpha_{i}\right|}\right)$. Notice that while replacing some $\alpha_{i}$ 's with the corresponding $-\alpha_{i}$ 's does not change the expansion in any important way, replacing some $d_{j}$ 's with the corresponding $-d_{j}$ 's is a more complicated matter and it may appear that allowing negative $d_{j}$ 's would result in pure products not attainable with only positive $d_{j}$ 's. We now explain why the new pure products that this would allow are not different in any important way from the ones attainable using only positive $d_{j}$ 's.

Theorem 5. Suppose $d_{1}, \ldots, d_{l} \in \mathbb{Z}$ are such that for $i=1, \ldots, n$, $\alpha_{i} \neq 0$ where $\alpha_{i}=\left|\sum_{j=1}^{l} p_{i, j} d_{j}\right|$. Then there exist $d_{1}^{\prime}, \ldots, d_{l}^{\prime} \in \mathbb{N}$ such that if $\alpha_{i}^{\prime}=\sum_{j=1}^{l} p_{i, j} d_{j}^{\prime}$ for $i=1, \ldots, n$, then $\left(\alpha_{1}^{\prime}, \ldots, \alpha_{n}^{\prime}\right)$ is a permutation of $\left(\alpha_{1}, \ldots, \alpha_{n}\right)$.

Proof. We define dot products by

$$
\left(x_{1}, \ldots, x_{k}\right) \cdot\left(y_{1}, \ldots, y_{k}\right):=\sum_{i=1}^{k} x_{i} y_{i} .
$$


Since some of the tuples in this proof represent vectors relative to the nonorthonormal basis $\Pi$, dot products involving these tuples do not satisfy the usual lengths-cosine relationship. So, for instance, saying that $\left(x_{1}, \ldots, x_{k}\right)$. $\left(y_{1}, \ldots, y_{k}\right)=0$ is not equivalent to saying that $x_{1} \pi_{1}+\ldots+x_{k} \pi_{k}$ is perpendicular to $y_{1} \pi_{1}+\ldots+y_{k} \pi_{k}$.

For $i=1, \ldots, n$, if $\left(p_{i, 1}, \ldots, p_{i, l}\right) \cdot\left(d_{1}, \ldots, d_{l}\right)>0$ then let $\varepsilon_{i}=1$; and if $\left(p_{i, 1}, \ldots, p_{i, l}\right) \cdot\left(d_{1}, \ldots, d_{l}\right)<0$ then let $\varepsilon_{i}=-1$. So we have the equation

$$
\left[\begin{array}{ccc}
\varepsilon_{1} p_{1,1} & \ldots & \varepsilon_{1} p_{1, l} \\
\varepsilon_{2} p_{2,1} & \ldots & \varepsilon_{2} p_{2, l} \\
\vdots & & \vdots \\
\varepsilon_{n} p_{n, 1} & \ldots & \varepsilon_{n} p_{n, l}
\end{array}\right]\left[\begin{array}{c}
d_{1} \\
\vdots \\
d_{l}
\end{array}\right]=\left[\begin{array}{c}
\alpha_{1} \\
\alpha_{2} \\
\vdots \\
\alpha_{n}
\end{array}\right] .
$$

For $i=1, \ldots, n$, let $r_{i}^{\prime}=\varepsilon_{i} r_{i}$. So for $i=1, \ldots, n, r_{i}^{\prime}=\sum_{j=1}^{l} \varepsilon_{i} p_{i, j} \pi_{j}$ and $r_{i}^{\prime}$ is a root in the root system $\Phi ; r_{i}^{\prime}$ is either $r_{i}$ or $-r_{i}$-whichever one has positive dot product with $\left(d_{1}, \ldots, d_{l}\right)$. That is, all the $r_{i}^{\prime}$ 's lie on the same side of the hyperplane of vectors having zero dot product with $\left(d_{1}, \ldots, d_{l}\right)$ (which are not necessarily perpendicular to $\left(d_{1}, \ldots, d_{l}\right)$ since the coordinates $\left(p_{i, 1}, \ldots, p_{i, l}\right)$ of each $r_{i}$ are with respect to the basis $\Pi$ which is not orthonormal). This means that $\left\{r_{i}^{\prime}: i \in I\right\}$ is a positive root system of the same type as $\Phi^{+}$— call this positive root system $\Phi^{\prime+}$. Denote the fundamental roots of $\Phi^{\prime+}$ by $\pi_{1}^{\prime}, \ldots, \pi_{l}^{\prime}$ and put $\Pi^{\prime}=\left\{\pi_{1}^{\prime}, \ldots, \pi_{l}^{\prime}\right\}$. For $i=1, \ldots, n$, let $\left(p_{i, 1}^{\prime}, \ldots, p_{i, l}^{\prime}\right)$ be the coordinates of $r_{i}^{\prime}$ relative to $\Pi^{\prime}$. That is, each $r_{i}^{\prime}=\sum_{j=1}^{l} p_{i, j}^{\prime} \pi_{j}^{\prime}$. Since $\Phi^{+}$and $\Phi^{\prime+}$ are positive root systems of the same type, we know that the matrices

$$
\left[\begin{array}{ccc}
p_{1,1} & \ldots & p_{1, l} \\
p_{2,1} & \cdots & p_{2, l} \\
\vdots & & \vdots \\
p_{n, 1} & \ldots & p_{n, l}
\end{array}\right] \quad \text { and } \quad\left[\begin{array}{ccc}
p_{1,1}^{\prime} & \ldots & p_{1, l}^{\prime} \\
p_{2,1}^{\prime} & \ldots & p_{2, l}^{\prime} \\
\vdots & & \vdots \\
p_{n, 1}^{\prime} & \ldots & p_{n, l}^{\prime}
\end{array}\right]
$$

have the same rows, but not necessarily in the same order. So we prove the theorem if we show that there exist $d_{1}^{\prime}, \ldots, d_{l}^{\prime} \in \mathbb{N}$ satisfying

$$
\left[\begin{array}{ccc}
p_{1,1}^{\prime} & \cdots & p_{1, l}^{\prime} \\
p_{2,1}^{\prime} & \cdots & p_{2, l}^{\prime} \\
\vdots & & \vdots \\
p_{n, 1}^{\prime} & \cdots & p_{n, l}^{\prime}
\end{array}\right]\left[\begin{array}{c}
d_{1}^{\prime} \\
\vdots \\
d_{l}^{\prime}
\end{array}\right]=\left[\begin{array}{c}
\alpha_{1} \\
\alpha_{2} \\
\vdots \\
\alpha_{n}
\end{array}\right]
$$

For $j=1, \ldots, l$, let $i_{j}^{\prime}$ be so that $r_{i_{j}^{\prime}}^{\prime}=\pi_{j}^{\prime}$. So for $j=1, \ldots, l, p_{i_{j}^{\prime}, 1}^{\prime}, \ldots, p_{i_{j}^{\prime}, l}^{\prime}$ are all 0 except for $p_{i_{j}^{\prime}, j}^{\prime}=1$. Hence, the only assignment of $d_{1}^{\prime}, \ldots, d_{l}^{\prime}$ that can satisfy the matrix multiplication above is to let each $d_{j}^{\prime}=\alpha_{i_{j}^{\prime}}$ 
since we require

$$
\begin{aligned}
\alpha_{i_{j}^{\prime}} & =\left(p_{i_{j}^{\prime}, 1}^{\prime}, \ldots, p_{i_{j}^{\prime}, l}^{\prime}\right) \cdot\left(d_{1}^{\prime}, \ldots, d_{l}^{\prime}\right) \\
& =(0, \ldots, 0,1,0, \ldots, 0) \cdot\left(d_{1}^{\prime}, \ldots, d_{j-1}^{\prime}, d_{j}^{\prime}, d_{j+1}^{\prime}, \ldots, d_{l}^{\prime}\right)=d_{j}^{\prime} .
\end{aligned}
$$

Notice that this means we are choosing $d_{1}^{\prime}, \ldots, d_{l}^{\prime}$ to be natural numbers.

Now we just need to verify that for $i=1, \ldots, n, \alpha_{i}=\sum_{j=1}^{l} p_{i, j}^{\prime} d_{j}^{\prime}$. This is actually fairly easy to see from equalities we have already established. The details are as follows. For $i=1, \ldots, n, r_{i}^{\prime}=\sum_{j=1}^{l} p_{i, j}^{\prime} \pi_{j}^{\prime}$, so $\left(p_{i, 1}^{\prime}, \ldots, p_{i, l}^{\prime}\right)$ $=\sum_{j=1}^{l} p_{i, j}^{\prime}\left(p_{i_{j}^{\prime}, 1}^{\prime}, \ldots, p_{i_{j}^{\prime}, l}^{\prime}\right)$ and $\left(\varepsilon_{i} p_{i, 1}, \ldots, \varepsilon_{i} p_{i, l}\right)=\sum_{j=1}^{l} p_{i, j}^{\prime}\left(\varepsilon_{i_{j}^{\prime}} p_{i_{j}^{\prime}, 1}, \ldots\right.$ $\left.\ldots, \varepsilon_{i_{j}^{\prime}} p_{i_{j}^{\prime}, l}\right)$. Now

$$
\begin{aligned}
\sum_{j=1}^{l} p_{i, j}^{\prime} d_{j}^{\prime} & =\left(p_{i, 1}^{\prime}, \ldots, p_{i, l}^{\prime}\right) \cdot\left(d_{1}^{\prime}, \ldots, d_{l}^{\prime}\right) \\
& =\left(\sum_{j=1}^{l} p_{i, j}^{\prime}\left(p_{i_{j}^{\prime}, 1}^{\prime}, \ldots, p_{i_{j}^{\prime}, l}^{\prime}\right)\right) \cdot\left(d_{1}^{\prime}, \ldots, d_{l}^{\prime}\right) \\
& =\sum_{j=1}^{l} p_{i, j}^{\prime}\left(\left(p_{i_{j}^{\prime}, 1}^{\prime}, \ldots, p_{i_{j}^{\prime}, l}^{\prime}\right) \cdot\left(d_{1}^{\prime}, \ldots, d_{l}^{\prime}\right)\right) \\
& =\sum_{j=1}^{l} p_{i, j}^{\prime} \alpha_{i_{j}^{\prime}}=\sum_{j=1}^{l} p_{i, j}^{\prime}\left(\left(\varepsilon_{i_{j}^{\prime}} p_{i_{j}^{\prime}, 1}, \ldots, \varepsilon_{i_{j}^{\prime}} p_{i_{j}^{\prime}, l}\right) \cdot\left(d_{1}, \ldots, d_{l}\right)\right) \\
& =\left(\sum_{j=1}^{l} p_{i, j}^{\prime}\left(\varepsilon_{i_{j}^{\prime}} p_{i_{j}^{\prime}, 1}, \ldots, \varepsilon_{i_{j}^{\prime}} p_{i_{j}^{\prime}, l}\right)\right) \cdot\left(d_{1}, \ldots, d_{l}\right) \\
& =\left(\varepsilon_{i} p_{i, 1}, \ldots, \varepsilon_{i} p_{i, l}\right) \cdot\left(d_{1}, \ldots, d_{l}\right)=\alpha_{i} . .
\end{aligned}
$$

In the next theorem, we choose the $d_{j}$ 's so that the restriction of $p$ to $\left\{\sum \Omega: \Omega \subseteq \Phi^{+}\right\}$is one-to-one. One can do this by choosing $d_{j}=$ $(C+1)^{j}$ where $C=\max \left\{c_{1}, \ldots, c_{l}\right\}$ and $\sum_{j=1}^{l} c_{j} \pi_{j}=\sum \Phi^{+}$, but we will use slightly smaller $d_{j}$ 's. Notice that when we make the restriction of $p$ to $\left\{\sum \Omega: \Omega \subseteq \Phi^{+}\right\}$one-to-one, the reason this makes the 2-norm small is that the coefficients in the expansion of the pure product we construct are each 0,1 , or -1 . Few methods are known for constructing such products.

Theorem 6. Let $c_{1}, \ldots, c_{l}$ be such that $\sum_{j=1}^{l} c_{j} \pi_{j}=\sum \Phi^{+}$. Let $d_{1}=1$ and assign $d_{2}, \ldots, d_{l}$ inductively by $d_{j}=d_{j-1}\left(c_{j-1}+1\right)$. (That is, $d_{j}=$ $\prod_{k=1}^{j-1}\left(c_{k}+1\right)$.) For $i=1, \ldots, n$, let $\alpha_{i}=\sum_{j=1}^{l} p_{i, j} d_{j}$. Then

$$
\left\|\prod_{i=1}^{n}\left(1-x^{\alpha_{i}}\right)\right\|_{2}=\sqrt{|W|} \text {. }
$$


Proof. Similarly to the proof of Theorem 4, we can say that

$$
\begin{aligned}
\left\|\prod_{i=1}^{n}\left(1-x^{\alpha_{i}}\right)\right\|_{2} & =\left\|\sum_{k=0}^{\infty} b(k) x^{k}\right\|_{2} \\
& =\left(\sum_{k=0}^{\infty}|b(k)|^{2}\right)^{1 / 2}=\left(\sum_{k=0}^{\infty}\left|\sum_{p(v)=k} a(v)\right|^{2}\right)^{1 / 2} .
\end{aligned}
$$

Observe by the following that if $k \in \mathbb{N} \cup\{0\}$ and $v \in\left\{\sum \Omega: \Omega \subseteq \Phi^{+}\right\}$are such that $p(v)=k$, then $v$ is uniquely determined. We know $v=\sum_{j=1}^{l} v_{j} \pi_{j}$ for some $v_{1}, \ldots, v_{l} \in \mathbb{N} \cup\{0\}$ such that each $v_{j} \leq c_{j}$. Since $d_{l}>\sum_{j=1}^{l-1} c_{j} d_{j}$, we know that $v_{l}=\left\lfloor k / d_{l}\right\rfloor$, and we find $v_{l-1}$ similarly after reducing $k$ modulo $d_{l}$, and so on. So for every $k \in \mathbb{N} \cup\{0\}$ we have $\left|\sum_{p(v)=k} a(v)\right| \in\{0,1\}$ and, hence, $\left|\sum_{p(v)=k} a(v)\right|^{2}=\left|\sum_{p(v)=k} a(v)\right|$. Together with Corollary 2, this tells us that

$$
\left(\sum_{k=0}^{\infty}\left|\sum_{p(v)=k} a(v)\right|^{2}\right)^{1 / 2}=\left(\sum_{k=0}^{\infty}\left|\sum_{p(v)=k} a(v)\right|\right)^{1 / 2}=|W|^{1 / 2} .
$$

Every indecomposable root system is of one of the following types. This table (except for the last column) is copied from Carter's book $\left[\right.$ C72, p. 43]. (A decomposable root system is one of the form $\bigcup_{i \in I} \Phi_{i}$ where $2 \leq|I|$ finite, each $\Phi_{i}$ is an indecomposable root system of rank $l_{i}$, and the $\Phi_{i}$ 's are embedded in $\left(\sum_{i \in I} l_{i}\right)$-space so that $r \perp s$ for all distinct $i, j \in I$ and all $r \in \Phi_{i}$ and $s \in \Phi_{j}$.)

Table 1. All indecomposable root systems

\begin{tabular}{cccc}
\hline Root system $\Phi$ & $n=\left|\Phi^{+}\right|$ & $|W|$ & $|W|$ \\
\hline$A_{l}(l \geq 1)$ & $\frac{1}{2} l(l+1)$ & $(l+1) !$ & $(\sqrt{2 n+1 / 4}+1 / 2) !$ \\
$B_{l}(l \geq 2)$ & $l^{2}$ & $2^{l} l !$ & $2^{\sqrt{n}} \sqrt{n !}$ \\
$C_{l}(l \geq 3)$ & $l^{2}$ & $2^{l} l !$ & ditto \\
$D_{l}(l \geq 4)$ & $l(l-1)$ & $2^{l-1} l !$ & $2^{\sqrt{n+1 / 4}-1 / 2}(\sqrt{n+1 / 4}+1 / 2) !$ \\
$G_{2}$ & 6 & 12 & \\
$F_{4}$ & 24 & $2^{7} \cdot 3^{2}$ & \\
$E_{6}$ & 36 & $2^{7} \cdot 3^{4} \cdot 5$ & \\
$E_{7}$ & 63 & $2^{10} \cdot 3^{4} \cdot 5 \cdot 7$ & \\
$E_{8}$ & 120 & $2^{14} \cdot 3^{5} \cdot 5^{2} \cdot 7$ &
\end{tabular}

Some readers may wonder about the less restrictive definition of root systems which allows a type known as $B C$. The root system $B C_{l}$ has $l(l+1)$ positive roots and the number corresponding to $|W|$ for our purposes is 
$(l+1) ! 2^{l}$. This makes $B C$-type root systems less useful to us than $B$ - and $C$-type root systems.

Carter [C72, Section 3.6] explicitly lists the roots of each root system in terms of an orthonormal basis. From these descriptions, we get the following $\alpha_{i}$ 's using the construction in Theorem 4. For $A_{l}$,

$$
\left\{\alpha_{i}: 1 \leq i \leq n\right\}=\left\{d_{i}+\ldots+d_{j}: 1 \leq i \leq j \leq l\right\} .
$$

For $B_{l}$,

$$
\begin{aligned}
\left\{\alpha_{i}: 1 \leq i \leq n\right\}= & \left\{d_{i}+\ldots+d_{j}: 1 \leq i \leq j \leq l\right\} \\
& \cup\left\{d_{i}+\ldots+d_{j-1}+2\left(d_{j}+\ldots+d_{l}\right): 1 \leq i<j \leq l\right\} .
\end{aligned}
$$

For $C_{l}$,

$$
\begin{aligned}
\left\{\alpha_{i}: 1 \leq\right. & i \leq n\} \\
= & \left\{d_{i}+\ldots+d_{j-1}: 1 \leq i<j \leq l\right\} \\
& \cup\left\{d_{i}+\ldots+d_{j-1}+2\left(d_{j}+\ldots+d_{l-1}\right)+d_{l}: 1 \leq i<j \leq l\right\} \\
& \cup\left\{2\left(d_{i}+\ldots+d_{l-1}\right)+d_{l}: 1 \leq i \leq l\right\} .
\end{aligned}
$$

For $D_{l}$,

$$
\begin{aligned}
& \left\{\alpha_{i}: 1 \leq i \leq n\right\} \\
& =\left\{d_{i}+\ldots+d_{j-1}: 1 \leq i<j \leq l\right\} \\
& \quad \cup\left\{d_{i}+\ldots+d_{j-1}+2\left(d_{j}+\ldots+d_{l-2}\right)+d_{l-1}+d_{l}: 1 \leq i<j \leq l\right\} .
\end{aligned}
$$

From Table 1, we see that using $B$-type root systems in Theorems 7 and 9 , we can construct $n$-factor pure products $\prod_{i=1}^{n}\left(1-x^{\alpha_{i}}\right)$ so that

$$
\left\|\prod_{i=1}^{n}\left(1-x^{\alpha_{i}}\right)\right\|_{1} \leq 2^{\sqrt{n}} \sqrt{n} ! \leq(2 \sqrt{n})^{\sqrt{n}}
$$

and

$$
\left\|\prod_{i=1}^{n}\left(1-x^{\alpha_{i}}\right)\right\|_{2}=\sqrt{2^{\sqrt{n}} \sqrt{n} !} \leq(2 \sqrt{n})^{\sqrt{n / 4}} .
$$

We now compare these bounds to those obtainable by methods in the literature.

3. Comparisons with old results. The relevant results in the literature are concerned mainly with the $\infty$-norms of pure products. The 1-norm, 2 -norm, and $\infty$-norm are related by the following easily verified inequalities (cf. [BI94]). For any polynomial $p(x)$,

$$
\frac{\|p(x)\|_{1}}{\sqrt{\operatorname{deg} p(x)+1}} \leq\|p(x)\|_{2} \leq\|p(x)\|_{\infty} \leq\|p(x)\|_{1} \leq\|p(x)\|_{2}^{2} .
$$


If one starts searching for pure products of small norm, one is likely to get the notion that for general $n \in \mathbb{N}, \prod_{i=1}^{n}\left(1-x^{i}\right)$ will have small norm, but this example turns out to be disappointing. According to Erdős \& Szekeres [ES58],

$$
\lim _{n \rightarrow \infty}\left(\left\|\prod_{i=1}^{n}\left(1-x^{i}\right)\right\|_{\infty}\right)^{1 / n}>1 .
$$

In other words, there is a constant $c>1$ so that for large $n$, the $\infty$-norm of the $n$-factor pure product $\prod_{i=1}^{n}\left(1-x^{i}\right)$ is roughly $c^{n}$. Atkinson [A61] and Dobrowolski [D79] showed by different methods that for any natural number $k$,

$$
\left\|\prod_{i=1}^{k-1}\left(1-x^{i}\right)^{k-i}\right\|_{\infty} \leq k^{k / 2} .
$$

(Borwein \& Ingalls [BI94] incorrectly gave the outer exponent in this pure product as $k-i-1$ instead of $k-i$.) Letting $n=k(k-1) / 2$, so that $k=1 / 2+\sqrt{2 n+1 / 4}$, we can say that this construction yields an $n$-factor pure product having $\infty$-norm at most $(1 / 2+\sqrt{2 n+1 / 4})^{1 / 4+\sqrt{n / 2+1 / 16}}$. If we allow the approximation $k \approx \sqrt{2 n}$, this bound simplifies to $\sqrt{2 n} \sqrt{n / 2}$. (In [BI94], Borwein \& Ingalls say incorrectly that $k \leq \sqrt{2 n}$.) This pure product can be constructed by using $A_{k-1}$ as the root system in Theorem 4 and letting $d_{1}=\ldots=d_{k-1}=1$. Borwein \& Ingalls [BI94] observed, essentially, that Dobrowolski's method achieves the same result for any values of the $d_{i}$ 's. The method relies on expressing the pure product in terms of a Vandermonde determinant and is not useful for root systems other than $A$-type.

The best bounds in the literature are only existence results - the authors do not give a construction of pure products witnessing their bounds, they only show that such pure products exist. Also, they do not express these bounds precisely, they only give the order. The exponent in Odlyzko's bound [O82] is the cube root $\sqrt[3]{n(\ln n)^{4}}$, improving on the square root in the exponent of $\sqrt{2 n} \sqrt{n / 2}$. Kolountzakis [K94] improved on Odlyzko's bound, getting an exponent of $\sqrt[3]{n}$. According to Odlyzko [O95], recent unpublished work of S. V. Konyagin and A. S. Belov makes a major improvement on these results, showing that there exists a constant $c$ so that $A_{\infty}(n) \leq e^{c(\ln n)^{4}}$ for all $n \in \mathbb{N}$. Our results will not approach these bounds, but our examples have the advantage of being explicit constructions.

If we construct a pure product $\prod_{i=1}^{n}\left(1-x^{\alpha_{i}}\right)$ from $A_{k-1}$ as in Theorem 4 , we know that $\left\|\prod_{i=1}^{n}\left(1-x^{\alpha_{i}}\right)\right\|_{1} \leq(\sqrt{2 n+1 / 4}+1 / 2)$ !. Furthermore, by choosing the $d_{i}$ 's as in Theorem 6 , we see that this bound is tight. Clearly, this bound is an improvement on the bound of $\sqrt{2 n}^{\sqrt{2 n}}$ resulting from Borwein \& Ingalls's inequalities $\|p\|_{1} \leq\|p\|_{\infty}^{2}$ and $\left\|\prod_{i=1}^{n}\left(1-x^{\alpha_{i}}\right)\right\|_{\infty} \leq$ 
$\sqrt{2 n} \sqrt{n / 2}$. However, one is really only interested in how small the smallest 1 -norm of an $n$-factor pure product can be. In this sense, we cannot improve on Dobrowolski's example in which $d_{1}=\ldots=d_{k-1}=1$ so that $\prod_{i=1}^{n}\left(1-x^{\alpha_{i}}\right)=\prod_{i=1}^{k}\left(1-x^{i}\right)^{k-i}$. Then, at least for $k \geq 4$,

$$
\begin{aligned}
\left\|\prod_{i=1}^{n}\left(1-x^{\alpha_{i}}\right)\right\|_{1} & \leq\left\|\prod_{i=1}^{k}\left(1-x^{i}\right)^{k-i}\right\|_{\infty} \sqrt{1+\operatorname{deg} \prod_{i=1}^{k}\left(1-x^{i}\right)^{k-i}} \\
& \leq k^{k / 2} \sqrt{1+\sum_{i=1}^{k} i(k-i)<k^{k / 2} \sqrt{\sum_{i=1}^{k}(k / 2)^{2}}} \\
& =k^{k / 2} \sqrt{k(k / 2)^{2}}=k^{k / 2} \frac{k^{3 / 2}}{2}=\frac{1}{2} k^{(k+3) / 2} \\
& \approx \sqrt{2 n}^{(\sqrt{2 n}+3) / 2} \approx \sqrt{2 n}^{\sqrt{n / 2}} .
\end{aligned}
$$

For the 2-norm, Theorem 6 improves on the literature. Using Borwein \& Ingalls's inequalities $\|p\|_{2} \leq\|p\|_{\infty}$ and $\left\|\prod_{i=1}^{n}\left(1-x^{\alpha_{i}}\right)\right\|_{\infty} \leq \sqrt{2 n}^{\sqrt{n / 2}}$, we get the bound $\left\|\prod_{i=1}^{n}\left(1-x^{\alpha_{i}}\right)\right\|_{2} \leq \sqrt{2 n}^{\sqrt{n / 2}}$. Using $A$-type root systems in Theorem 6 to construct the same pure product, we get the small improvement

$$
\left\|\prod_{i=1}^{n}\left(1-x^{\alpha_{i}}\right)\right\|_{2}=\sqrt{(\sqrt{2 n+1 / 4}+1 / 2) !} .
$$

Better than this, using $B$ - or $C$-type root systems in Theorem 6 , we get (for $n \geq 36$ )

$$
\left\|\prod_{i=1}^{n}\left(1-x^{\alpha_{i}}\right)\right\|_{2}=\sqrt{2^{\sqrt{n}} \sqrt{n} !}<\sqrt{\sqrt{n}^{\sqrt{n}}}=\sqrt{n}^{\sqrt{n / 4}} .
$$

Using Theorem 6 with $B$ - or $C$-type root systems is better than any other method known for constructing pure products of small 2-norm.

We now consider pure products having particular numbers of factors. In Table 2, each $p_{n}$ is an $n$-factor pure product of minimal known 1-norm. A table of pure products of minimal known 1-norm appeared in Borwein \& Ingalls's paper [BI94]. We found a few errors and corrections for their table, but it seems pointless to report these now since Borwein has since improved on these results. The results in Table 2 are Borwein's [B96] for $n=15,24$, 28 , and 30 through 70 . Some of the other pure products in this table are also Borwein's [B96], but they are not actually improvements on other results in terms of the size of the 1-norm. Other results are in [BI94], [M97], and [M96]. We include 2-norms in the table since, as far as we know, no search 
has been made specifically for pure products of small 2-norm. We use two kinds of ellipsis in the table. The single ellipsis "..." means that every number between the bounds appears exactly once, and the double ellipsis ":::" means that every odd number between the bounds appears exactly once.

Table 2. Exponents of pure products of least known 1-norm

\begin{tabular}{|c|c|c|c|}
\hline$n$ & $\left\|p_{n}\right\|_{1}$ & $\left\|p_{n}\right\|_{2}^{2}$ & Exponents of $p_{n}$ \\
\hline 3 & 6 & 6 & {$[1,2,3]$} \\
\hline 4 & 8 & 10 & {$[1,2,3,4]$} \\
\hline 5 & 10 & 10 & {$[1,3,4,5,7]$} \\
\hline 6 & 12 & 12 & {$[1,2,3,4,5,7]$} \\
\hline 7 & 16 & 16 & {$[1,2,3,4,5,7,11]$} \\
\hline 8 & 16 & 16 & {$[2,3,5,7,8,11,13,19]$} \\
\hline 9 & 20 & 20 & {$[2,3,5,7,8,11,13,17,19]$} \\
\hline 10 & 24 & 24 & {$[1, \ldots, 5,7,9,11,13,17]$} \\
\hline 11 & 28 & 28 & {$[1,2,3,5,7,8,9,11,13,17,19]$} \\
\hline 12 & 36 & 40 & {$[1, \ldots, 9,11,13,17]$} \\
\hline 13 & 48 & 52 & {$[1, \ldots, 9,11,13,17,19]$} \\
\hline 14 & 56 & 56 & {$[1,3, \ldots, 7,10,11,13,16,17,19,23,29]$} \\
\hline 15 & 52 & 52 & {$[1,3, \ldots, 7,9,10,11,13,16,17,19,23,29]$} \\
\hline 16 & 60 & 68 & {$[1, \ldots, 11,:::, 19,23]$} \\
\hline 17 & 68 & 68 & {$[1, \ldots, 7,9,10,11,13,14,16,17,19,23,29]$} \\
\hline 18 & 84 & 94 & {$[1, \ldots, 11,13,14,16,17,19,22,23]$} \\
\hline 19 & 100 & 108 & {$[1, \ldots, 11,:::, 25,29]$} \\
\hline 20 & 116 & 132 & {$[1, \ldots, 11,:::, 27,31]$} \\
\hline 21 & 130 & 142 & {$[1, \ldots, 11,:::, 31]$} \\
\hline 22 & 140 & 152 & {$[1, \ldots, 9,:::, 33,37]$} \\
\hline 23 & 156 & 168 & {$[1, \ldots, 11,:::, 33,37]$} \\
\hline 24 & 192 & 208 & {$[1, \ldots, 11,13,14,15,:::, 31,35,37]$} \\
\hline 25 & 188 & 204 & {$[1, \ldots, 11,:::, 37,41]$} \\
\hline 26 & 228 & 256 & {$[1, \ldots, 11,:::, 41]$} \\
\hline 27 & 276 & 324 & {$[1, \ldots, 13,:::, 41]$} \\
\hline 28 & 292 & 350 & {$[1, \ldots, 13,:::, 41,47]$} \\
\hline 29 & 392 & 696 & {$[1,1,2,2,3, \ldots, 27]$} \\
\hline 30 & 396 & 760 & {$[1,1,2,2,3,3,4, \ldots, 27]$} \\
\hline 31 & 414 & 714 & {$[1,1,2,2,3, \ldots, 29]$} \\
\hline 32 & 456 & 852 & {$[1,1,2,2,3,3,4, \ldots, 29]$} \\
\hline 33 & 482 & 930 & {$[1,1,2,2,3,3,4, \ldots, 29,31]$} \\
\hline 34 & 516 & 974 & {$[1,1,2,2,3,3,4, \ldots, 31]$} \\
\hline 35 & 604 & 1248 & {$[1,1,2,2,3,3,4, \ldots, 31,33]$} \\
\hline 36 & 616 & 1200 & {$[1,1,2,2,3,3,4, \ldots, 33]$} \\
\hline
\end{tabular}


Table 2 (cont.)

\begin{tabular}{|c|c|c|c|}
\hline$n$ & $\left\|p_{n}\right\|_{1}$ & $\left\|p_{n}\right\|_{2}^{2}$ & Exponents of $p_{n}$ \\
\hline 37 & 688 & 1440 & {$[1,1,2,2,3,3,4, \ldots, 33,35]$} \\
\hline 38 & 664 & 1338 & {$[1,1,2,2,3,3,4, \ldots, 35]$} \\
\hline 39 & 740 & 1460 & {$[1,1,2,2,3,3,4, \ldots, 35,37]$} \\
\hline 40 & 804 & 1564 & {$[1,1,2,2,3,3,4, \ldots, 37]$} \\
\hline 41 & 908 & 1976 & {$[1,1,2,2,3,3,4, \ldots, 37,39]$} \\
\hline 42 & 924 & 1996 & {$[1,1,2,2,3,3,4, \ldots, 39]$} \\
\hline 43 & 952 & 2032 & {$[1,1,2,2,3,3,4, \ldots, 39,41]$} \\
\hline 44 & 1028 & 2144 & {$[1,1,2,2,3,3,4, \ldots, 41]$} \\
\hline 45 & 1132 & 2516 & {$[1,1,2,2,3,3,4, \ldots, 41,43]$} \\
\hline 46 & 1176 & 2866 & {$[1,1,2,2,3,3,4, \ldots, 43]$} \\
\hline 47 & 1254 & 2910 & {$[1,1,2,2,3,3,4,4,5, \ldots, 43]$} \\
\hline 48 & 1288 & 3180 & {$[1,1,2,2,3,3,4,4,5, \ldots, 44]$} \\
\hline 49 & 1484 & 3692 & {$[1,1,2,2,3,3,4,4,5, \ldots, 45]$} \\
\hline 50 & 1604 & 4400 & {$[1,1,2,2,3,3,4,4,5, \ldots, 46]$} \\
\hline 51 & 1632 & 4416 & {$[1,1,2,2,3,3,4,4,5,5,6, \ldots, 45,47]$} \\
\hline 52 & 1740 & 4586 & {$[1,1,2,2,3,3,4,5,5,6, \ldots, 47,49]$} \\
\hline 53 & 1602 & 3686 & {$[1,1,2,2,3,3,4,4,5,5,6, \ldots, 47,49]$} \\
\hline 54 & 1784 & 4616 & {$[1,1,2,2,3,3,4,4,5,5,6,7,7,8, \ldots, 47,49]$} \\
\hline 55 & 1916 & 4924 & {$[1,1,2,2,3,3,4,4,5,5,6, \ldots, 50]$} \\
\hline 56 & 2144 & 5928 & {$[1,1,2,2,3,3,4,4,5,5,6, \ldots, 51]$} \\
\hline 57 & 2188 & 3988 & {$[1,2,2,3, \ldots, 25, \ldots: 33,34,35,:: ., 85]$} \\
\hline 58 & 2352 & 4828 & {$[1,2,2,3, \ldots, 27, \ldots: 3,33,34,35,:::, 85]$} \\
\hline 59 & 2256 & 4068 & {$[1,2,2,3, \ldots, 25,::: 33,34,35,:::, 89]$} \\
\hline 60 & 2456 & 4780 & {$[1,2,2,3, \ldots, 27,::: 33,34,35,:::, 89]$} \\
\hline 61 & 2742 & 5486 & {$[1,2,2,3, \ldots, 27,::: 33,34,35,:::, 89,101]$} \\
\hline 62 & 2760 & 5164 & {$[1,2,2,3, \ldots, 27,:::, 93,101]$} \\
\hline 63 & 2992 & 5940 & {$[1,2,2,3,4,4,5, \ldots, 27,:::, 93,101]$} \\
\hline 64 & 3232 & 6884 & {$[1,2,2,3, \ldots, 29,:::, 93,101,107]$} \\
\hline 65 & 3130 & 6190 & {$[1,2,2,3, \ldots, 29,:::, 95,101,107]$} \\
\hline 66 & 3108 & 5904 & {$[1,2,2,3, \ldots, 29,:::, 97,101,107]$} \\
\hline 67 & 3508 & 7272 & {$[1,2,2,3, \ldots, 29,:: ., 37,38,39,::: 97,101,107]$} \\
\hline 68 & 3864 & 8224 & {$[1,2,2,3, \ldots, 29,:::, 97,101,107,109,111]$} \\
\hline 69 & 4464 & 10968 & {$[1,2,2,3, \ldots, 29,::: 63,64,65,::: 97,101,107,109,111]$} \\
\hline 70 & 4592 & 11444 & {$[1,2,2,3, \ldots, 29,:: ., 45,46,47, \ldots: 6,63,64,65,:: ., 97,101,107,109,111]$} \\
\hline
\end{tabular}

Theorem 4 gives an upper bound on the 1-norm of any pure product constructed from root systems as described. It seems likely that good choices of the $d_{i}$ 's in this construction will actually provide 1-norms much smaller than this upper bound. The most ambitious goal is to see if we can use the 
root system constructions to match the smallest known 1-norms, and for this purpose it seems best to begin by comparing the bounds from Theorem 4 with the best known 1-norms to see if any are already close. Since Borwein (and Ingalls's) results do not go up to 120 -factor pure products, we take $p_{120}$ to be $\left(p_{60}\right)^{2}$.

Table 3. Comparing best known norms to norms from root systems

\begin{tabular}{rlrrr}
\hline$n$ & System & $|W|$ & $\left\|p_{n}\right\|_{1}$ & $\left\|p_{n}\right\|_{2}^{2}$ \\
\hline 1 & $A_{1}$ & 2 & 2 & 2 \\
3 & $A_{2}$ & 6 & 6 & 6 \\
6 & $A_{3}$ & 24 & 12 & 12 \\
10 & $A_{4}$ & 120 & 24 & 24 \\
15 & $A_{5}$ & 720 & 52 & 52 \\
4 & $B_{2}$ & 8 & 8 & 10 \\
9 & $B_{3}, C_{3}$ & 48 & 20 & 20 \\
16 & $B_{4}, C_{4}$ & 384 & 60 & 68 \\
25 & $B_{5}, C_{5}$ & 3840 & 188 & 204 \\
12 & $D_{4}$ & 192 & 36 & 40 \\
20 & $D_{5}$ & 1920 & 116 & 132 \\
30 & $D_{6}$ & 23,040 & 396 & 760 \\
6 & $G_{2}$ & 12 & 12 & 12 \\
24 & $F_{4}$ & 1152 & 192 & 208 \\
36 & $E_{6}$ & 51,840 & 616 & 1200 \\
63 & $E_{7}$ & $2,903,040$ & 2992 & 5940 \\
120 & $E_{8}$ & $696,729,600$ & $1,440,480$ & $1,025,261,796$ \\
\hline
\end{tabular}

We see from Table 3 that constructions according to Theorem 4 always match the best known 1-norms when the root system used is $A_{1}, A_{2}, B_{2}$, or $G_{2}$. This does not hold in other cases but it would be interesting to know if we can always match the best known (or even best possible) results using the construction in Theorem 4 if we choose suitable values for the $d_{i}$ 's.

From [M97], we know that the least possible 1-norm of a 9-factor pure product is 20 and that there are only five ways (up to multiplication of all the exponents by a constant) to achieve this - the list of exponents $\left[\alpha_{1}, \ldots, \alpha_{9}\right]$ must be one of the following:

$$
\begin{aligned}
& {[1,2,3,4,5,7,9,11,13],} \\
& {[1,2,3,5,7,8,9,11,13],} \\
& {[1,2,3,5,7,8,11,13,19],} \\
& {[1,4,5,6,7,9,11,13,17],} \\
& {[2,3,5,7,8,11,13,17,19] .}
\end{aligned}
$$


The only root systems that produce 9 factors in the construction in Theorem 4 are $B_{3}$ and $C_{3}$. Can $B_{3}$ or $C_{3}$ produce any of the 9-exponent lists above using this construction? No. The details of the tedious case examinations appear in [M96].

For a given number of roots, decomposable root systems tend to have larger Weyl groups than indecomposable root systems do, so one might think it hopeless to ask if any of these 9-factor pure products of minimal 1-norm can be constructed from a decomposable root system. In fact, it is trivial to construct any pure product $\prod_{i=1}^{n}\left(1-x^{\alpha_{i}}\right)$ from a decomposable root system - just use the root system constructed from $n$ copies of $A_{1}$ and let $d_{i}=\alpha_{i}$ for $i=1, \ldots, n$. However, any resulting smallness of the norm will not be due to properties of root systems so this is not an enlightening answer.

The list of exponents of $p_{10}$ is $[1,2,3,4,5,7,9,11,13,17]$ and $\left\|p_{10}\right\|_{1}=24$. The only root system that produces 10 -factor pure products is $A_{4}$, and $A_{4}$ does not produce $p_{10}$, but it does come tantalisingly close. Using $A_{4}$ in the construction in Theorem 4 and letting $\left(d_{1}, d_{2}, d_{3}, d_{4}\right)=(4,1,2,10)$, we get $\left[\alpha_{1}, \ldots, \alpha_{10}\right]=[1,2,3,4,5,7,10,12,13,17]$, which differs from the list in question only in that 9 and 11 are replaced by 10 and 12 . Unfortunately, this tiny change has a substantial effect on the 1-norm, upping it from 24 to 60 .

The preceding examples discourage hope that root systems are always the best way to construct pure products of small norm, but perhaps if we look at Table 3 comparing the best known results with the worst possible by root systems, we can find a largish example where these quantities are not far apart. We seem to have such an example in $F_{4}$ which produces 24 -factor pure products of 1 -norm at most $2^{7} \cdot 3^{2}=1152$, while the least known 1-norm of a 24 -factor pure product is 192 . Because of the freedom we have in choosing $d_{1}, d_{2}, d_{3}, d_{4}$, we might expect to be able to produce a pure product using $F_{4}$ in Theorem 4 which has 1-norm much less than the worst case of 1152 . In fact, a substantial improvement is possible, but not to the point of breaking any records as far as we can see. The least 1-norm we have found for a 24 -factor pure product constructed from $A_{4}$ by the method in Theorem 4 is 328 and the exponents $\left[\alpha_{i}\right]$ in the pure product are

$$
[1,2,3,4,6,7,8,9,10,16,20,22,23,24,25,26,28,29,30,31,32,34,38,54] \text {. }
$$

Insofar as 120-factor pure products have been investigated at all in the literature, the construction in Theorem 4 using $E_{8}$ is an improvement, at least in terms of producing a small 2-norm. The norms for $p_{120}$ in Table 3 are based on taking $p_{120}=\left(p_{60}\right)^{2}$, which is about the best we can extrapolate from the literature. (Borwein \& Ingalls [BI94] only searched for pure products of small 1-norm up to 100 factors, and the largest good one 
they found had only 60 factors. Borwein [B96] decided to only keep track of $n$-factor pure products having 1-norm less than $n^{2}$, of which he found examples only up to $n=70$.) Another 120 -factor pure product from the literature is that of Atkinson [A61] and Dobrowolski [D79] which is the $A_{15}$ construction using $d_{1}=\ldots=d_{15}=1$. This yields 1 -norm $10,642,137,208$ and 2-norm square root of $672,106,633,395,797,608$, both much worse than the norms from $E_{8}$. Furthermore, remember that $|W|$ given in Table 3 for $E_{8}$ is an upper bound on the 1-norm and that with appropriate choices of $d_{1}, \ldots, d_{8}$, we expect that a much smaller 1 -norm is possible.

In Theorem 6, we see that with an appropriate choice of the $d_{i}$ 's in Theorem 4 , we can use any root system with $n$ positive roots and associated Weyl group $W$ to construct an $n$-factor pure product having $|W|$ coefficients 1 or -1 and all other coefficients 0 . One might wonder if there is always a choice of the $d_{i}$ 's so that this construction produces a polynomial in which all coefficients are \pm 1 (and none are zero). Considering $A_{2}$, we can see quickly that the answer is no. The pure product we get from $A_{2}$ is $\left(1-x^{d_{1}}\right)\left(1-x^{d_{2}}\right)\left(1-x^{d_{1}+d_{2}}\right)=1-x^{d_{1}}-x^{d_{2}}+x^{2 d_{1}+d_{2}}+x^{d_{1}+2 d_{2}}-x^{2 d_{1}+2 d_{2}}$. To get the coefficient of $x$ to be \pm 1 , we need $d_{1}=1$ and $d_{2} \geq 2$ (assuming, without loss of generality, that $d_{2} \geq d_{1}$ ). Then, to get the coefficient of $x^{2}$ to be \pm 1 , we need $d_{2}=2$. The resulting pure product is $1-x-x^{2}+x^{4}+x^{5}-x^{6}$ in which $x^{3}$ has coefficient 0 .

\section{References}

[A61] F. V. Atkinson, On a problem of Erdös and Szekeres, Canad. Math. Bull. 1 (1961), 7-12.

[B96] P. Borwein, e-mail communication.

[BI94] P. Borwein and C. Ingalls, The Prouhet-Tarry-Escott Problem revisited, Enseign. Math. 40 (1994), 3-27.

[C72] R. W. Carter, Simple Groups of Lie Type, Wiley, 1972.

[D79] E. Dobrowolski, On a question of Lehmer and the number of irreductible factors of a polynomial, Acta Arith. 34 (1979), 391-401.

[ES58] P. Erdös and G. Szekeres, On the product $\prod_{k=1}^{n}\left(1-z^{\alpha_{k}}\right)$, Acad. Serbe Sci. Publ. Inst. Math. 12 (1958), 29-34.

[K94] M. N. Kolountzakis, Probabilistic and Constructive Methods in Harmonic Analysis and Additive Number Theory, Ph.D. dissertation, Stanford University, 1994.

[M72] I. G. MacDonald, Affine root systems and Dedekind's $\eta$-function, Invent. Math. 15 (1972), 91-143.

[M96] R. Maltby, Pure Product Polynomials of Small Norm, Ph.D. dissertation, Simon Fraser University, 1996.

[M97] —, Pure product polynomials and the Prouhet-Tarry-Escott Problem, Math. Comp. (1997), to appear.

[M94] S. Maltby, Some optimal results related to the PTE Problem, preprint. 
[O82] A. M. Odlyzko, Minima of cosine sums and maxima of polynomials on the unit circle, J. London Math. Soc. (2) 26 (1982), 412-420.

[O95] - personal communication.

Centre for Experimental and Constructive Mathematics

Simon Fraser University

Burnaby, British Columbia

Canada, V5A 1S6

E-mail: maltby@cecm.sfu.ca

Received on 5.4.1996

and in revised form on 12.12.1996 